ఠ

\title{
Five-year alendronate treatment outcome in older postmenopausal Japanese women with osteoporosis or osteopenia and clinical risk factors for fractures
}

This article was published in the following Dove Press journal:

Therapeutics and Clinical Risk Management

24 September 2009

Number of times this article has been viewed

Jun Iwamoto'

Atsushi Miyata ${ }^{2}$

Yoshihiro Sato ${ }^{3}$

Tsuyoshi Takeda'

Hideo Matsumoto'

'Institute for Integrated Sports

Medicine, Keio University

School of Medicine, Tokyo, Japan;

${ }^{2}$ Department of Internal Medicine,

Yahata Clinic, Tokyo, Japan;

${ }^{3}$ Department of Neurology, Mitate

Hospital, Fukuoka, Japan
Correspondence: Jun Iwamoto Institute for Integrated Sports Medicine, Keio University School of Medicine, 35 Shinanomachi, Shinjuku-ku,

Tokyo 160-8582, Japan

Tel +8I 33353 I2I I

$\mathrm{Fax}+81333529467$

Email jiwamoto@sonata.plala.or.jp

\begin{abstract}
A retrospective study was conducted to evaluate the outcome of treatment with alendronate (ALN) for 5 years in postmenopausal Japanese women with an increased risk of fractures. Forty postmenopausal Japanese women with osteoporosis or osteopenia and clinical risk factors for fractures (mean age: 75.4 years) were analyzed; 33 patients were treated with alendronate and 7 were treated with alfacalcidol (ALF, controls) in an outpatient clinic run by general practitioners. The metacarpal bone mineral density (BMD) measured using a computed $\mathrm{X}$-ray densitometer, urinary levels of cross-linked N-terminal telopeptides of type I collagen (NTX), and serum levels of alkaline phosphatase (ALP) were monitored during the 5-year treatment period. The urinary NTX and serum ALP levels decreased significantly in the ALN group, compared with the ALF group. The metacarpal BMD was sustained in the ALN group but decreased significantly in the ALF group; the difference between these two groups was also significant. The present study evaluated the outcome of treatment with ALN for 5 years in postmenopausal Japanese women with osteoporosis or osteopenia and clinical risk factors for fractures. ALN successfully suppressed bone turnover and sustained the metacarpal BMD over the 5-year period of treatment in postmenopausal Japanese women with an increased risk of fractures.
\end{abstract}

Keywords: alendronate, osteoporosis, osteopenia, risk factors for fractures, postmenopausal women

\section{Background}

Osteoporosis most commonly affects postmenopausal women, placing them at a significant risk for fractures. Alendronate (ALN) and alfacalcidol (ALF) are widely used for the treatment of postmenopausal osteoporosis in Japan. The fracture intervention trial showed the antifracture efficacy of ALN for vertebral, nonvertebral, hip, and writs fractures in postmenopausal women with osteoporosis. ${ }^{1,2}$ A recent systematic review analyzing 11 randomized controlled trials (RCTs) representing 12068 women confirms both clinically important and statistically significant reductions in vertebral, nonvertebral, hip, and wrist fractures for secondary prevention (gold level evidence). ${ }^{3}$ However, current global evidence suggests that ALF does not help to prevent vertebral, nonvertebral, and hip fractures in postmenopausal women with osteoporosis. ${ }^{4}$ In postmenopausal Japanese women with osteoporosis, some evidence suggests that ALF helps to prevent vertebral fractures. ${ }^{5}$ A head-to-head trial showed that ALN was more effective than ALF for preventing vertebral fractures. ${ }^{6,7}$ Thus, ALN is regarded as a first-line drug and ALF is regarded as a second-line drug for the treatment of osteoporosis in Japan. 
The long-term efficacy of anti-fracture drugs needs to be established. Bone et $a l^{8}$ reported their experience using ALN to treat postmenopausal Western women with osteoporosis for a period of 10 years. Ten years of treatment with ALN produced an increase in the bone mineral density (BMD) of $13.7 \%$ at the lumbar spine, $10.3 \%$ at the trochanter, $5.4 \%$ at the femoral neck, and $6.7 \%$ at the total proximal femur, compared with the baseline values. Safety data, including fractures and stature, did not suggest that prolonged treatment resulted in any loss of benefit. Thus, the therapeutic effects of alendronate were sustained, and the drug was well tolerated over a 10 -year period. In Japan, although the anti-fracture efficacy of treatment with ALN for 3 years against vertebral fractures has been reported in postmenopausal women with osteoporosis, ${ }^{7}$ the outcome of longer-term ( $>3$ years) treatment with ALN remains to be established.

Based on the Japanese guideline for the prevention and treatment of osteoporosis, ${ }^{9}$ patients with osteoporosis or osteopenia and clinical risk factors for fractures should be treated using anti-fracture drugs. The presently reported retrospective study was conducted by Japanese general practitioners to evaluate the outcome of treatment with ALN for 5 years in postmenopausal Japanese women with osteoporosis or osteopenia and clinical risk factors for fractures. The primary endpoint was metacarpal $\mathrm{BMD}$, and the secondary endpoints were biochemical markers.

\section{Subjects and methods Subjects}

Forty postmenopausal Japanese women (age range: 60 to 93 years, mean age: 75.4 years at the beginning of treatment) who had been treated with ALN or ALF for over 5 years were recruited at the outpatient clinic of Yahata Clinic (Tokyo, Japan) during the 3-month period between February 1 and April 30, 2009. The inclusion criteria were postmenopausal women with osteoporosis or osteopenia and clinical risk factors for fractures at the start of treatment. The clinical risk factors for fractures included current smoking, maternal history of hip fractures, alcohol consumption $\geq 2$ units daily, age $\geq 75$ years, leanness (body mass index $\leq 18.5 \mathrm{~kg} / \mathrm{m}^{2}$ ), and history of steroid use. The exclusion criteria were histories of reflux esophagitis, gastric or duodenal ulcer, gastrectomy, or bone diseases, including primary hyperparathyroidism, hyperthyroidism, Cushing syndrome, multiple myeloma, rheumatoid arthritis, and osteogenesis imperfecta.

All the patients had been diagnosed as having osteoporosis according to the Japanese diagnostic criteria. ${ }^{10,11}$ Namely, patients with a BMD $<70 \%$ of the young adult mean (YAM) or of $70 \%$ to $80 \%$ of the YAM along with a history of osteoporotic fractures were diagnosed as having osteoporosis. Patients with a BMD of $70 \%$ to $80 \%$ of the YAM and without any history of osteoporotic fracture were diagnosed as having osteopenia. A preliminary screening included a medical history, physical examination, plain $\mathrm{X}$-rays of the thoracic and lumbar spine, metacarpal BMD measurement, and blood and urinary biochemical tests including serum calcium, phosphorus, and alkaline phosphatase (ALP) and urinary cross-linked N-terminal telopeptides of type I collagen (NTX).

Of the 40 patients, 33 had been treated with ALN ( $5 \mathrm{mg}$ daily or $35 \mathrm{mg}$ weekly) and 7 had been treated with ALF ( $1 \mu \mathrm{g}$ daily, controls) for over 5 years. Basically, ALN treatment was recommended to all patients, but those who refused it were treated with ALF, because ALN is a first line drug whereas ALF is a second line drug. The doses indicated in the parentheses above are the doses used in Japan for the treatment of postmenopausal women with osteoporosis and have been recognized as being safe and effective. ${ }^{5,12}$ Daily ALN and ALF treatment were available throughout the study period, but weekly ALN treatment only became available after October 2006. All the patients in the ALN group had been treated with daily ALN and then switched to weekly ALN just after October 2006. The effects of daily and weekly ALN treatments on BMD and bone turnover markers as well as the incidence of side effects were reported to be similar in postmenopausal Japanese women with osteoporosis..$^{13}$ The subjects did not receive either elementary calcium or natural vitamin D supplementation.

The urinary levels of NTX were measured at 3 months after the start of treatment based on the results of our previous study. ${ }^{14}$ Urinary NTX measurement at 3 months after the start of ALN treatment is important and enough to monitor its effect in the treatment of osteoporosis. ${ }^{14}$ The serum levels of calcium, phosphorus, and ALP and the metacarpal BMD were measured every 6 months after the start of treatment. The outcomes of treatment with ALN or ALF for 5 years were compared.

Informed consent was obtained from each patient with regard to the use of their clinical data in the present report. The present study was approved by the Ethics Committee of Yahata Clinic.

\section{Assessment of vertebral fractures}

Plain lateral X-ray films of the thoracic and lumbar spine were obtained at baseline to detect evidence of morphometric vertebral fractures. According to the Japanese criteria, 
a vertebral fracture was defined according to the vertebral height on lateral X-ray films. ${ }^{10,11}$ Briefly, the vertebral height was measured at the anterior $(\mathrm{A})$, central $(\mathrm{C})$, and posterior (P) parts of the vertebral body, and the presence of a vertebral fracture was confirmed when (1) a reduction in the vertebral height of more than $20 \%(\mathrm{~A}, \mathrm{C}$, and $\mathrm{P}$ ) compared with the height of the adjacent vertebrae was observed, (2) the C/A or $\mathrm{C} / \mathrm{P}$ was less than 0.8 , or (3) the $\mathrm{A} / \mathrm{P}$ was less than 0.75 . The assessment for vertebral fractures was performed at the T4-L4 level.

\section{Measurement of metacarpal BMD}

An anteroposterior view radiograph of bilateral hands and an aluminum step wedge (20 steps, $1 \mathrm{~mm}$ per step) was obtained for each subject and was used as a standard. The BMD of the middle portion of the second metacarpus on the left hand was measured using a computed X-ray densitometer (Teijin, Tokyo, Japan). The densitometer calculated the BMD based on the pattern expressed as graduations on the aluminum step wedge, and the BMD was expressed as the thickness of the aluminum equivalent ( $\mathrm{mmAl}$ ) showing a corresponding $\mathrm{X}$-ray absorption. For these measurements, the coefficients of variation $(\mathrm{CV}, 100 \times$ standard deviation $(\mathrm{SD}) /$ mean $)$ for the intraobserver error (based on 10 consecutive measurements by 5 persons), the daily change error (based on measurements performed on 10 consecutive days), and the X-ray picturing error (based on 10 exposures) were all within $1.3 \%$ to $3.8 \%$.

\section{Statistical analysis}

Data were expressed as the mean \pm SD in the tables and the mean $\pm 95 \%$ confidence interval $(\mathrm{CI})$ in the figures. Comparisons of the baseline characteristics between the 2 groups were performed using an unpaired t-test. The significance of longitudinal changes in the parameters in a group was determined using a one-way analysis of variance (ANOVA) with repeated measurements. Longitudinal changes in the parameters were compared between the two groups using a two-way ANOVA with repeated measurements. The percentage of women with fractures at baseline was compared between the two groups using the Fisher exact test. All statistical analyses were performed using the Stat View-J5.0 program. A significance level of $P<0.05$ was used for all the comparisons.

\section{Results}

\section{Characteristics of the study subjects}

\section{at the start of treatment}

Table 1 shows the baseline characteristics of the study subjects. In the ALN group $(\mathrm{n}=33), 19$ patients had osteoporosis and 14 had osteopenia and clinical risk factors for fractures ( $n=4$ for age $\geq 75$ years; $n=3$ for leanness, $n=2$ each for history of steroid use (just transient use), current smoking, and maternal history of hip fractures; $\mathrm{n}=1$ for alcohol consumption $\geq 2$ units daily). In the ALF group $(n=7)$, on the other hand, 5 patients had osteoporosis and 2 had osteopenia and clinical risk factors for fractures $(n=1$ each for age $\geq 75$ years and leanness).

Table I Characteristics of study subjects

\begin{tabular}{|c|c|c|c|}
\hline & ALN group $(n=33)$ & ALF group $(n=7)$ & $P$ values \\
\hline Age (years) & $75.5 \pm 7.8$ & $74.9 \pm 9.5$ & NS \\
\hline Height (m) & $1.49 \pm 0.07$ & $1.52 \pm 0.06$ & NS \\
\hline Body weight $(\mathrm{kg})$ & $49.3 \pm 8.8$ & $49.3 \pm 8.3$ & NS \\
\hline Body mass index $\left(\mathrm{kg} / \mathrm{m}^{2}\right)$ & $22.2 \pm 3.1$ & $21.1 \pm 2.3$ & NS \\
\hline Metacarpal BMD (mmAl) & $1.92 \pm 0.21$ & $1.86 \pm 0.19$ & NS \\
\hline \%YAM of metacarpal BMD (\%) & $70.2 \pm 7.6$ & $67.7 \pm 6.8$ & NS \\
\hline Serum calcium $(\mathrm{mg} / \mathrm{dL})$ & $9.1 \pm 0.3$ & $9.0 \pm 0.4$ & NS \\
\hline Serum phosphorus (mg/dL) & $3.6 \pm 0.5$ & $3.8 \pm 0.6$ & NS \\
\hline Serum ALP (IU/L) & $262 \pm 74$ & $231 \pm 69$ & NS \\
\hline Urinary NTX (nmol BCE/mmol Cr) & $67.5 \pm 24.9$ & $55.5 \pm 12.3$ & NS \\
\hline $\begin{array}{l}\text { Number (\%) of women with prevalent } \\
\text { vertebral fractures }\end{array}$ & $9(27.3)$ & I (I4.3) & NS \\
\hline $\begin{array}{l}\text { Number (\%) of women with history of } \\
\text { nonvertebral fractures }\end{array}$ & $3(9.1)$ & I (I4.3) & NS \\
\hline
\end{tabular}

Notes: Data are expressed as the mean \pm SD. Data comparison between the 2 groups was performed using an unpaired t-test. The percentage of patients with fractures were compared using the Fisher exact test.

Abbreviations: ALN, alendronate;ALF, alfacalcidol; BMD, bone mineral density; YAM, young adult mean;ALP, alkaline phosphatase; NTX, cross linked N-terminal telopeptides of type I collagen; BCE, bone collagen equivalent; $\mathrm{Cr}$, creatinine; NS, not significant. 
No significant differences in any of the baseline characteristics including anthropometry, metacarpal BMD, biochemical markers and the number of patients with prevalent vertebral fractures or a history of nonvertebral fractures were observed between the two groups. The mean levels of urinary NTX in both groups suggested high turnover osteoporosis (the normal range for Japanese women: 9.3 to $54.3 \mathrm{nmol}$ bone collagen equivalents $[\mathrm{BCE}] / \mathrm{mmol} \mathrm{Cr}) .{ }^{15}$

\section{Changes in biochemical markers and metacarpal BMD}

Figure 1 shows the changes in the biochemical markers. The urinary NTX levels were reduced to the normal range for Japanese women $(9.3 \text { to } 54.3 \mathrm{nmol} \mathrm{BCE} / \mathrm{mmol} \mathrm{Cr})^{11}$ after 3 months of treatment, and the serum ALP levels remained within the normal range (135 to $310 \mathrm{IU} / \mathrm{L}$ ) during the 5-year period in the ALN groups. However, the urinary NTX and serum ALP levels did not change significantly in the ALF group. The mean change rates of the urinary NTX levels after 3 months of treatment in the ALN and ALF groups were $-43.6 \%$ and $+13.3 \%$, respectively. The respective change rates of the serum ALP levels after 5 years of treatment were $-18.0 \%$ and $+0.8 \%$. The urinary NTX and serum ALP levels decreased significantly in the ALN group, compared with in the ALF group (Table 2). However, the changes in the serum calcium and phosphorus levels did not differ significantly between the two groups.

Figure 2 shows the changes in metacarpal BMD. Metacarpal BMD was sustained in the ALN group $(+0.4 \%$ at 5 years) but decreased significantly in the ALF group $(-5.4 \%$ at 5 years); the difference between the 2 groups was also significant (Table 2).

\section{Discussion}

The results of RCTs to test the effects of short-term (1 to 3 years) treatment with ALN on BMD, bone turnover markers, and fracture incidence have been reported in postmenopausal Japanese women with osteoporosis. ${ }^{6,7,12,13}$ ALN reduces bone turnover markers, increases lumbar and hip BMD, and prevents vertebral fractures. However, the outcome of long-term ( $>3$ years) treatment with ALN has not been previously reported. The objective of the present
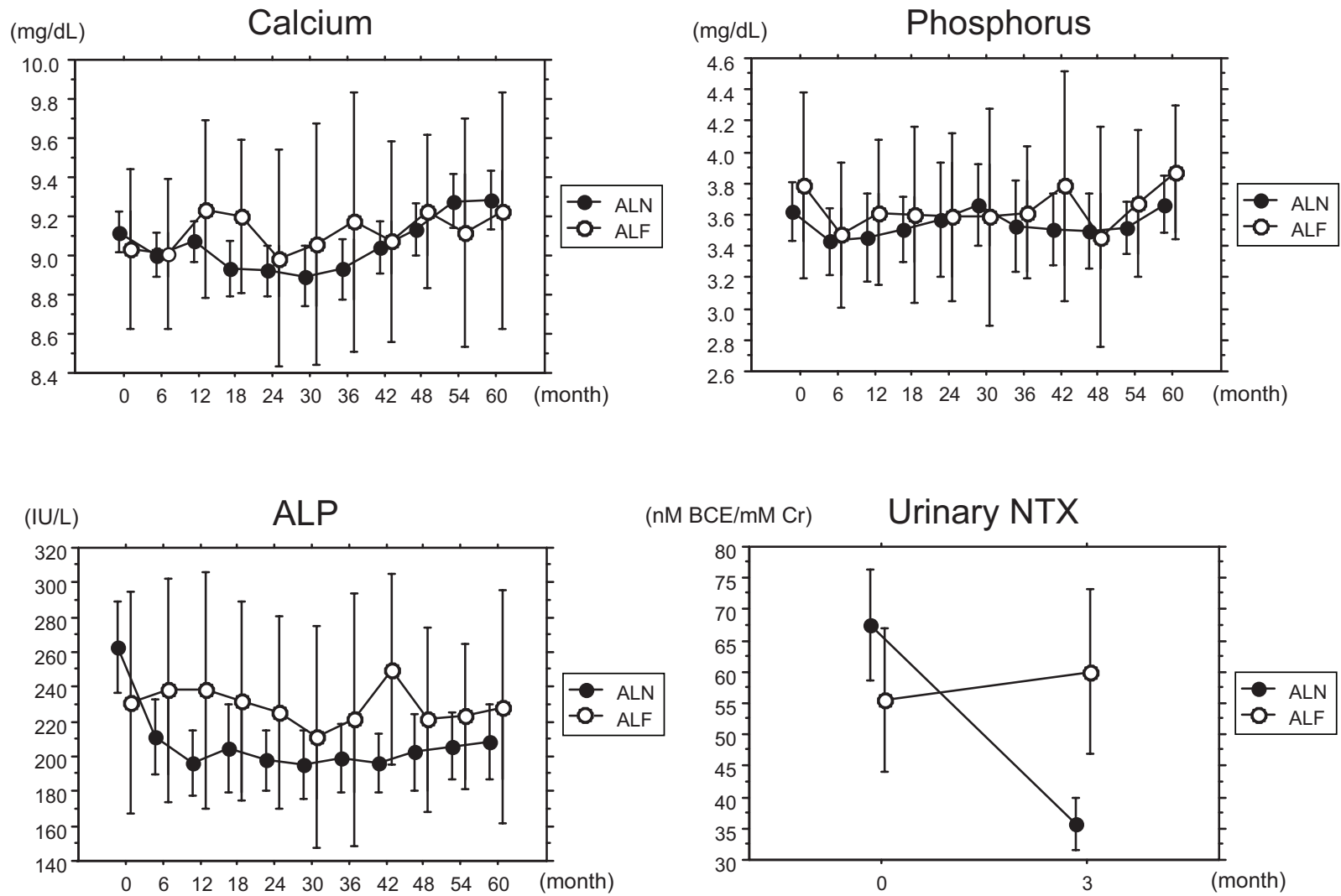

(nM BCE/mM Cr) Urinary NTX

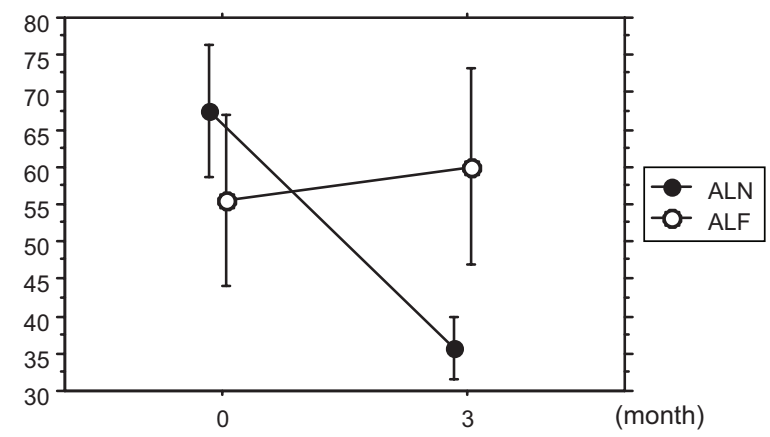

Figure I Changes in biochemical markers.

Notes: Data are expressed as the mean $\pm 95 \%$ confidence interval $(\mathrm{Cl})$. The results of statistical analyses are shown in Table 2.

Abbreviations: ALN, alendronate;ALF, alfacalcidol;ALP, alkaline phosphatase; NTX, cross linked N-terminal telopeptides of type I collagen. 
Table 2 Results of ANOVA with repeated measurements

\begin{tabular}{lllll}
\hline & $\begin{array}{l}\text { One-way } \\
\text { ANOVA }\end{array}$ & & & $\begin{array}{l}\text { Two-way } \\
\text { ANOVA }\end{array}$ \\
\cline { 2 - 3 } \cline { 5 - 5 } \cline { 5 - 5 } & ALN & ALF & & ALN vs ALF \\
\hline Serum calcium & $<0.0001$ & NS & & NS \\
Serum phosphorus & NS & NS & NS \\
Serum ALP & $<0.0001$ & NS & & 0.0199 \\
Urinary NTX & $<0.0001$ & NS & & 0.0001 \\
Metacarpal BMD & NS & 0.0026 & 0.0011 \\
\hline
\end{tabular}

Notes: $P$ values are presented. The significance of longitudinal changes in biochemical markers and metacarpal BMD in the ALN or ALF group was determined using a one-way ANOVA with repeated measurements. Differences in longitudinal changes in biochemical markers and metacarpal BMD were compared using a 2-way ANOVA with repeated measurements.

Abbreviations: ANOVA, analysis of variance; ALN, alendronate; ALF, alfacalcidol; BMD, bone mineral density; ALP, alkaline phosphatase; NTX, cross linked N-terminal telopeptides of type I collagen; NS, not significant.

retrospective study was to evaluate the outcome of treatment with ALN for 5 years in postmenopausal women with osteoporosis or osteopenia and clinical risk factors for fractures according to the clinical practices of Japanese general practitioners. The present study confirmed that ALN successfully suppressed bone turnover and sustained the metacarpal BMD over a period of 5 years in postmenopausal Japanese women with an increased risk of fractures. However, because withdrawn patients were not included among the subjects in the present study, the 5-year safety of ALN treatment, including its adverse effects, remains uncertain.

In the present study, postmenopausal Japanese women with osteopenia and clinical risk factors for fractures were included among the study subjects. According to the Japanese guidelines for the prevention and treatment of osteoporosis, ${ }^{9}$ not only patients with osteoporosis, but also those with osteopenia and clinical risk factors for fractures should be treated using anti-fracture drugs. Clinical risk factors for fractures included current smoking, a maternal history of hip fractures, and alcohol consumption $\geq 2$ units daily. ${ }^{9}$ In addition, the following factors were also included: age $\geq 75$ years, leanness, and history of steroid use; a previous report in Japanese subjects indicated that these factors were risk factors for fractures independent of the BMD. ${ }^{9}$ Siris et $\mathrm{al}^{16}$ reported that using a peripheral measurement device, $82 \%$ of postmenopausal women with fractures had T scores better than -2.5 and that a strategy for reducing the overall fracture incidence would likely require lifestyle changes and a targeted effort to identify and develop treatment protocols for women with less severe low BMD values who nonetheless had an increased risk for future fractures. Thus, it may be reasonable to treat postmenopausal women with osteopenia and clinical risk factors for fractures.

In postmenopausal Japanese women with osteoporosis, studies have shown that ALN (5 mg daily or $35 \mathrm{mg}$ weekly) decreases the urinary NTX ( $-45 \%$ at 3 months) and serum ALP (about $-20 \%$ at 3 years) levels and increases the lumbar $\operatorname{BMD}(+9.2 \%$ at 3 years $) .{ }^{7,13}$ Another study showed that treatment with ALN for 1 year increased the lumbar BMD but only sustained the metacarpal BMD. ${ }^{17}$ In the present study, treatment with ALN sustained the metacarpal BMD for 5 years after reducing the urinary levels of NTX $(-43.6 \%$ at 3 months) and the serum levels of ALP ( $-18.0 \%$ at 5 years). The urinary NTX levels were reduced to within the normal range for Japanese women after 3 months of treatment, while the serum ALP levels remained within the normal range throughout the 5-year period of the present study. We evaluated serum NTX (the normal range for Japanese women: 7.5 to $16.5 \mathrm{nmol} \mathrm{BCE} / \mathrm{L})^{15}$ after 5 years treatment as not described in the subjects and methods section. The serum levels of NTX (nmol BCE/L, mean \pm SD) at 5 years were $10.2 \pm 2.8$ for the ALN group and $17.6 \pm 19.4$ for the ALF group with a significant difference between the 2 groups $(P<0.0001$ by an unpaired t-test), suggesting the sustained effect of ALN over the 5-year period of treatment.

Dual-energy X-ray absorptiometry (DXA) is used to measure lumbar, femoral neck and forearm BMD, while computed X-ray densitometry can be used to measure metacarpal BMD. DXA-based BMD measurements at the lumbar spine and femoral neck are useful for predicting the risk of osteoporotic fractures in Japan. ${ }^{9-11}$ However, because DXA machines are very expensive, not all clinics and hospitals are equipped with such devices. Therefore, computed X-ray densitometry is usually used in Japanese clinics and hospitals that do not have a DXA machine. The efficacy of ALN for increasing the lumbar BMD, as determined using DXA, has been confirmed in postmenopausal Japanese women with osteoporosis. ${ }^{6,7,12,13}$

The effect of ALN on the BMD of the middle part of the metacarpus, which is composed of cortical bone, is poor. Tucci et $a{ }^{18}$ reported the differential effects of ALN on the BMD at various skeletal sites. Their results suggested that appendicular bones may be less responsive to ALN than axial bones in postmenopausal women with osteoporosis; distal sites of appendicular bones may be less responsive than proximal sites; and smaller appendicular bones may be less responsive than larger appendicular bones. Second, the ratio of cancellous to cortical bone differs among skeletal sites. The lumbar spine is very rich in cancellous bone, whereas 
(mmAl) Metacarpal BMD

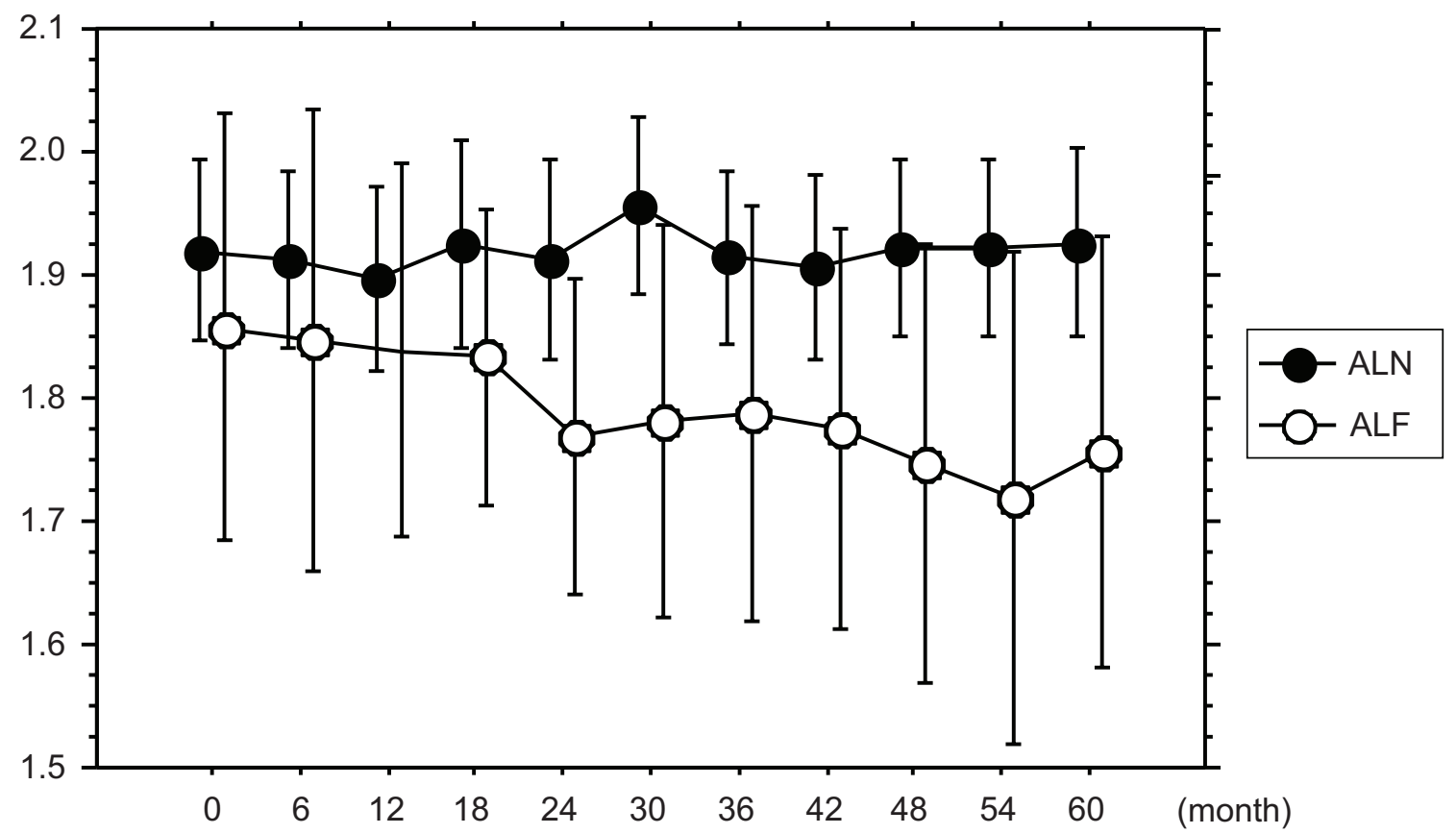

Figure 2 Changes in metacarpal BMD.

Notes: Data are expressed as the mean $\pm 95 \%$ confidence interval $(\mathrm{Cl})$. The results of statistical analyses are shown in Table 2 .

Abbreviations: ALN, alendronate; ALF, alfacalcidol; BMD, bone mineral density.

the middle portion of the metacarpus consists of cortical bone. Because the acceleration of bone remodeling is widely observed on the trabecular surface rather than the endocortical and intracortical Haversian canal surfaces, cancellous bone may be more responsive to ALN than cortical bone. However, the results of the present study did not suggest that prolonged treatment resulted in any loss of benefit regarding metacarpal BMD.

Shiraki et a ${ }^{19}$ reported that 6 months of treatment with ALF reduced the serum levels of parathyroid hormone and bone turnover markers in elderly Japanese women with high bone turnover osteoporosis. They also reported that treatment with ALF for 5 years increased the BMD $(+6 \%)$ of the radius (peripheral cortical bone site) in Japanese patients with osteoporosis. ${ }^{20}$ Orimo et $\mathrm{al}^{5}$ showed that treatment with ALF for one year slightly increased the lumbar BMD $(+0.65 \%)$ and reduced the incidence of vertebral fractures in postmenopausal Japanese women with osteoporosis. These results suggest that ALF may reduce bone turnover, increase the BMD, and prevent vertebral fractures in postmenopausal Japanese women with osteoporosis. In the present study, however, long-term treatment with ALF did not affect the urinary NTX and serum ALP levels or the metacarpal BMD. The reason for this discrepancy between the results of previous studies and the present results remains uncertain, although the small number of study subjects in the present study might be an important factor. Nevertheless, the metacarpal BMD seems to have been sustained during the first 18 months after the start of ALF treatment (Figure 2). Thus, the short-term effect of ALF on BMD might be satisfactory. In addition, the BMD of the radius may be more responsive to ALF than that of the metacarpus.

The most notable limitation of the present study is its retrospective and head-to-head design with a small sample size only in postmenopausal Japanese women with an increased risk for fractures. First, some of the results, especially those of the ALF group, might be biased and the evidence level of our results is low. Second, the results might only be applicable for postmenopausal Japanese women with an increased risk for fractures who consult Japanese general practitioners. Third, the safety of ALN, including its side effects, remains uncertain, although the safety of treatment with ALN for 10 years has been established in postmenopausal Western women with osteoporosis. ${ }^{8}$ Fourth, there was not sufficient statistical power to allow a conclusion to be made regarding the effect of ALN on the fracture incidence. Thus, a prospective randomized 
head-to-head trial with a sufficient number of subjects is needed to confirm the results of the present study.

In conclusion, the present retrospective study, which was performed by Japanese general practitioners, reported the outcome of treatment with ALN for 5 years in postmenopausal women with osteoporosis or osteopenia and clinical risk factors for fractures. ALN successfully suppressed bone turnover and sustained the metacarpal BMD for 5 years in postmenopausal Japanese women with an increased risk of fractures.

\section{Disclosures}

The authors report no funding sources or conflict of interest in this work.

\section{References}

1. Black DM, Cummings SR, Karpf DB, et al. Randomised trial of effect of alendronate on risk of fracture in women with existing vertebral fractures. Lancet. 1996;348:1535-1541.

2. Cummings SR, Black DM, Thompson DE, et al. Effect of alendronate on risk of fracture in women with low bone density but without vertebral fractures: results from the Fracture Intervention Trial. JAMA. 1998;280:2077-2082.

3. Wells GA, Cranney A, Peterson J, et al. Alendronate for the primary and secondary prevention of osteoporotic fractures in postmenopausal women. Cochrane Database Syst Rev. 2008;23(1):CD001155.

4. The WHO Scientific Group. Prevention and management of osteoporosis. World Health Organ Tech Rep Ser. 2003;921:86-109.

5. Orimo H, Shiraki M, Hayashi Y, et al. Effects of 1 alpha-hydroxyvitamin D3 on lumbar bone mineral density and vertebral fractures in patients with postmenopausal osteoporosis. Calcif Tissue Int. 1994;54:370-376.

6. Kushida K, Shiraki M, Nakamura T, et al. The efficacy of alendronate in reducing the risk for vertebral fracture in Japanese patients with osteoporosis:a randomized, double-blind, active-controlled, doubledummy trial. Current Therapeutic Research. 2002;63:9:606-620.

7. Kushida K, Shiraki M, Nakamura T, et al. Alendronate reduced vertebral fracture risk in postmenopausal Japanese women with osteoporosis: a 3-year follow-up study. J Bone Miner Metab. 2004;22:462-468.
8. Bone HG, Hosking D, Devogelaer JP, et al; Alendronate Phase III Osteoporosis Treatment Study Group. Ten years' experience with alendronate for osteoporosis in postmenopausal women. N Engl J Med. 2004;350:1189-1199.

9. Orimo H. Japanese guideline for prevention and treatment of osteoporosis. Life Science. 2006.

10. Orimo H, Sugioka Y, Fukunaga M, et al. Diagnostic criteria of primary osteoporosis. J Bone Miner Metab. 1998;16:139-150.

11. Orimo H, Hayashi Y, Fukunaga M, et al. Diagnostic criteria for primary osteoporosis: year 2000 revision. J Bone Miner Metab. 2001;19: 331-337.

12. Shiraki M, Kushida K, Fukunaga M, et al. A double-masked multicenter comparative study between alendronate and alfacalcidol in Japanese patients with osteoporosis. Osteoporos Int. 1999;10:183-192.

13. Uchida S, Taniguchi T, Shimizu T, et al. Therapeutic effects of alendronate $35 \mathrm{mg}$ once weekly and $5 \mathrm{mg}$ once daily in Japanese patients with osteoporosis: a double-blind, randomized study. J Bone Miner Metab. 2005;23:382-388.

14. Iwamoto J, Takeda T, Sato Y, Uzawa M. Early changes in urinary cross-linked N-terminal telopeptides of type I collagen level correlate with one-year response of lumbar bone mineral density to alendronate in Japanese postmenopausal women with osteoporosis. J Bone Miner Metab. 2005;23:238-242.

15. Nishizawa Y, Nakamura T, Ohta $\mathrm{H}$, et al; Committee on the Guidelines for the Use of Biochemical Markers of Bone Turnover in Osteoporosis Japan Osteoporosis Society. Guidelines for the use of biochemical markers of bone turnover in osteoporosis (2004). J Bone Miner Metab. 2005;23:97-104.

16. Siris ES, Chen YT, Abbott TA, et al. Bone mineral density thresholds for pharmacological intervention to prevent fractures. Arch Intern Med. 2004;164:1108-1112.

17. Iwamoto J, Takeda T, Sato Y, et al. Effects of alendronate on metacarpal and lumbar bone mineral density, bone resorption, and chronic back pain in postmenopausal women with osteoporosis. Clin Rheumatol. 2004;23:383-389.

18. Tucci JR, Tonino RP, Emkey RD, et al. Effect of three years of oral alendronate treatment in postmenopausal women with osteoporosis. Am J Med. 1996;101:488-501.

19. Shiraki M, Fukuchi M, Kiriyama T, et al. Alfacalcidol reduces accelerated bone turnover in elderly women with osteoporosis. J Bone Miner Metab. 2004;22:352-359.

20. Shiraki M, Ito H, Orimo $H$. The ultra long-term treatment of senile osteoporosis with 1 alpha-hydroxyvitamin $\mathrm{D}_{3}$. Bone Miner. 1993;20:223-234.
Therapeutics and Clinical Risk Management

\section{Publish your work in this journal}

Therapeutics and Clinical Risk Management is an international, peerreviewed journal of clinical therapeutics and risk management, focusing on concise rapid reporting of clinical studies in all therapeutic areas, outcomes, safety, and programs for the effective, safe, and sustained use of medicines. This journal is indexed on PubMed Central, CAS,

\section{Dovepress}

EMBase, Scopus and the Elsevier Bibliographic databases. The manuscript management system is completely online and includes a very quick and fair peer-review system, which is all easy to use. Visit http://www.dovepress.com/testimonials.php to read real quotes from published authors. 